\title{
Correspondence
}

Editor: Ian Pullen

Contents: Old versus new ECT/Daily living programme/Enhancement of recovery from psychiatric illness by methylfolate/Schizophrenia following prenatal exposure to influenza epidemics between 1939 and 1960/Psychotherapy in non-Western cultures/ Lithium augmentation in antidepressant-resistant patients/Who benefits from ECT?/Do benzodiazepines interfere with the action of ECT?/Drug therapy of post-traumatic stress disorder/Acute transient stress-induced hallucinations in soldiers/Child psychiatry in the 20th century/Sex differences in the familial risk of schizophrenia: data from Rüdin's study/Absence of prion protein mutation in bipolar manic-depressive patients/Amnesia in fugue states neurological or psychogenic basis/The distorting influence of time/Iron Maiden's déjà vu/Hypnotised lobsters/Currie's complaint?

\section{Old versus new ECT}

SIR: Scott et al (Journal, March 1992, 160, 360-364) report a randomised trial comparing efficacy of traditional and modern electroconvulsive therapy (ECT) devices. Eleven out of 17 patients $(65 \%)$ receiving constant-voltage ECT and 9 out of 14 (64\%) receiving constant-current ECT attained 'recovery' defined by an HRSD score of less than 8 . The authors stated that "... the likelihood of recovery ... [was] virtually identical in the two groups ... the policy of bilateral suprathreshold modern ECT monitored by EEG [electroencephalogram] is as efficacious as traditional ECT". They must have given some thought to sample size, since they state it "was too small to attempt to identify any potential subsets of depressed patients who may respond preferentially to ECT". A reader's misgivings about the small size of the whole study could be allayed by the authors' attention to this issue.

The British Medical Journal requires, in my opinion rightly, confidence intervals to support the main conclusions of a study. Use of confidence intervals at this instance reveals clearly how little one can be sure that the difference is negligible. A 95\% CI for 11 out of 17 is $38 \%$ to $86 \%$; the corresponding interval for 9 out of 14 is $35 \%$ to $87 \%$ (Lentner,
1982). The difference in recovery rates is determined only to within a margin of error of $34 \%$ : an approximate $95 \% \mathrm{CI}$ for the difference is $-33 \%$ to $+34 \%$. It is clear that a clinically important difference, in favour of either of the methods of administering ECT, is not ruled out.

LENTNER, C. (ed.) (1982) Geigy Scientific Tables. Vol. 2. Introduction to Statistics, Statistical Tables, Mathematical Formulae. 8th ed., pp. 89-104. Basle: Ciba-Geigy.

R. G. NEWCOMBE

Department of Medical Computing and Statistics University of Wales College of Medicine

Heath Park

Cardiff CF4 4XN

AUTHOR'S REPLY: The critique of the interpretation of our findings rests on several assumptions. Firstly, it is assumed that we assess the clinical efficacy of ECT upon recovery rate alone. This is not so, and Newcombe makes no mention of the other measures of clinical outcome after the course of ECT. Secondly, it is assumed that we base our interpretation on the results of this randomised comparative study alone. This is not so; for example, the recovery rate after brief-pulse ECT was identical $(64 \%)$ to that in an earlier study that used the same definition of recovery (Scott et al, 1986). Thirdly, the critique takes no account of the existing literature that we cited. When comparative studies of traditional and brief-pulse ECT are designed to take account of stimulus intensity, no difference in clinical efficacy is found. Finally, the extreme limits of the $95 \%$ confidence intervals for the recovery rates are quoted to suggest that we may have failed to detect a "clinically important difference". Gardner \& Altman (1989), the statistical advisers to the British Medical Journal, stated "regardless of the width of the confidence interval, the sample estimate is the best indicator of the population value". Table 1 showed that none of the estimates (means) of clinical outcome after ECT (final HRSD score, final Montgomery \& Asberg score, number of ECT prescribed, and recovery rate) showed any difference of clinical significance. 